\title{
Caos, organização e criatividade: revisão integrativa sobre as Redes de Atenção à Saúde
}

\author{
Chaos, organization and creativity: \\ integrative review on Health Care Networks
}

Luís Fernando Nogueira Tofani (https://orcid.org/0000-0002-1092-2450) ${ }^{1}$

Lumena Almeida Castro Furtado (https://orcid.org/0000-0001-7897-9739) ${ }^{1}$

Cristian Fabiano Guimarães (https://orcid.org/0000-0003-3101-634X) ${ }^{2}$

Deize Graziele Conceição Ferreira Feliciano (https://orcid.org/0000-0002-4407-6379) ${ }^{1}$

Gabriela Rodrigues da Silva (https://orcid.org/0000-0001-9378-4219) ${ }^{1}$

Larissa Maria Bragagnolo (https://orcid.org/0000-0002-6643-8465) ${ }^{1}$

Rosemarie Andreazza (https://orcid.org/0000-0002-3332-2183) ${ }^{1}$

Arthur Chioro (https://orcid.org/0000-0001-7184-2342) ${ }^{1}$

$\overline{{ }^{1} \text { Departamento de Medicina }}$ Preventiva, Escola Paulista de Medicina, Universidade Federal de São Paulo. R. Botucatu 740 , Vila Clementino. 04023-062 São Paulo SP Brasil. luis. tofani@gmail.com

${ }^{2}$ Faculdade Meridional. Passo Fundo RS Brasil.

\begin{abstract}
Since 2010, the organization of the Brazilian Unified Health System has as its main model the Health Care Networks, based on the Integrated Health Service Networks recommended by the Pan American Health Organization. This study aims to analyze the scientific production on Health Care Networks the integrative literature review method. The research was conducted in databases using the descriptors: Health Care Network and its counterparts in Portuguese and Spanish. A total of 27 articles were selected, including conceptual studies, case reports, implementation analyses and evaluation studies. The analysis of the publications evidenced five cores of meaning: the Health Care Network paradigm; Primary Care as network coordinator; regionalization, networks and regional governance; network care; and challenges for implementation. The results of this review point to two central questions: how to overcome the fragmentation of care, ensuring integrality, composing health care networks from distinct theoretical conceptions? And how can the production of care networks impact inter-federative relations, the financing process, the access to the system and the quality of health care processes?
\end{abstract}

Key words Health Care Networks, Integrated Health Service Networks, Health Systems
Resumo A partir de 2010 a organização do Sistema Único de Saúde tem como principal modelo as Redes de Atenção à Saúde (RAS), fundamentadas nas Redes Integradas de Serviços de Saúde preconizadas pela Organização Panamericana de Saúde. Este estudo tem como objetivo analisar a produção científica sobre as RAS através do método de revisão integrativa da literatura. A pesquisa deu-se em bases de dados utilizando-se dos descritores: Rede de Atenção à Saúde e seus congêneres em inglês e espanhol. Foram selecionados 27 artigos incluindo estudos conceituais, relatos de casos, análises de implementação e estudos de avaliação. A análise das publicações evidenciou cinco núcleos de sentido: O paradigma das RAS; A Atenção Básica como coordenadora das RAS; Regionalização, redes e governança regional; O cuidado em rede; $e$, Desafios para implementação das RAS. Os resultados dessa revisão apontam para duas questões centrais: Como superar a fragmentação do cuidado, garantindo a integralidade, compondo as redes de atenção à saúde a partir de concepções teóricas distintas? E, como a produção das redes de cuidado pode impactar nas relações interfederativas, no processo de financiamento, no acesso ao sistema e na qualidade dos processos de cuidado em saúde? Palavras-chave Redes de Atenção à Saúde, Redes Integradas de Serviços de Saúde, Sistemas de Saúde 


\section{Introdução}

No Brasil, o Sistema Único de Saúde (SUS) foi concebido e é organizado por meio de uma rede regionalizada e hierarquizada de serviços e ações, que tem por objetivo garantir a saúde como um direito constitucional. Em seu processo de construção, inúmeras normativas têm induzido arranjos organizativos a partir de diretrizes como a descentralização, a municipalização, a regionalização, a participação da sociedade e a gestão interfederativa, caracterizando inovações na estrutura do Estado e na administração pública do país ${ }^{1}$. Apesar do atual cenário político nacional de inflexão de políticas públicas e da supressão de direitos, o que se observa é a materialização das diretrizes organizacionais da descentralização e da regionalização, o que propiciou avanços e também desafios na estruturação do sistema de saúde ${ }^{2}$.

A partir de 2010 o modelo de organização do SUS passou a ter como principal referência as Redes de Atenção à Saúde (RAS), modelo fundamentado nas Redes Integradas de Serviços de Saúde (RISS) preconizadas pela Organização Pan-Americana de Saúde ${ }^{3}$. Este modelo vem sendo utilizado em países em processo de transição demográfica e epidemiológica onde predominam condições e agravos crônicos ${ }^{4}$, vem sendo apresentado como alternativa à fragmentação dos sistemas de saúde ${ }^{5}$ e indica a centralidade da atenção primária nas redes ${ }^{6-8}$.

As principais justificativas para a implantação das RAS são a necessidade de organização de sistemas para dar respostas à tríplice carga de doenças - infectocontagiosas, crônicas e causas externas - e a obtenção de melhores resultados econômicos, epidemiológicos e de integralidade do cuidado em saúde 4 . Para tanto, propugna-se a organização de redes constituídas por diversos pontos de atenção à saúde, com centralidade da atenção primária, sistemas logísticos e de apoio?. Segundo Silva e Magalhães Junior ${ }^{10}$, a implantação das RAS justifica-se também pela necessidade de cuidados continuados em saúde para pessoas com condições crônicas, como estratégia para garantia da integralidade e pela perspectiva da economia de escala e de escopo.

Cecílio ${ }^{11}$ já propunha uma revisão do modelo hierarquizado da pirâmide, constituída por atenção primária, secundária e terciária, apontando a necessidade do sistema de saúde ser organizado a partir da lógica do que seria mais importante para cada usuário, e assim oferecer a tecnologia certa, no espaço certo e na ocasião mais adequada. Também Hartz e Contandriopoulos ${ }^{12}$ apre- sentaram a proposta de um "sistema sem muros", em que se eliminariam as barreiras de acesso entre os diversos níveis de atenção, em resposta às necessidades de saúde nos âmbitos local e regional. Por fim, a normativa legal que instituiu as Redes de Atenção à Saúde no Brasil propôs um modelo poliárquico de sistema constituído por diferentes pontos de atenção à saúde e pelas ligações que os comunicam, com o objetivo de se obter melhores resultados epidemiológicos e de integralidade do cuidado em saúde ${ }^{13}$.

Desta forma, a indução pelo Ministério da Saúde pela implantação das Redes de Atenção à Saúde, a partir de 2011, representou uma nova etapa de organização do SUS, com o objetivo de garantir a integralidade do cuidado e produzir mudanças nos modos de produção do cuidado à saúde através de redes temáticas prioritárias, que foram denominadas como Rede Cegonha, Rede de Urgência e Emergência, Rede de Atenção Psicossocial, Rede de Saúde da Pessoa com Deficiência e Rede de Atenção às Doenças Crônicas ${ }^{14}$.

Considerando a relevância da Política de Redes de Atenção à Saúde, torna-se imprescindível a realização de estudos que possam analisar e disseminar as publicações que tomaram o tema como objeto de investigação, visando ampliar a visibilidade e motivar a realização de novas produções científicas acerca das RAS para subsidiar os processos de formulação, implementação e avaliação de políticas de saúde no SUS. Peiter et al. ${ }^{15}$ analisaram a tendência de investigação sobre as RAS em teses e dissertações brasileiras, a partir da base de dados do Catálogo de Teses e Dissertações da Coordenação de Aperfeiçoamento de Pessoal de Nível Superior (CAPES), entretanto não há estudo de revisão da literatura publicada em periódicos sobre a temática. Ante o exposto, surgiu a motivação para o desenvolvimento deste estudo, que tem como objetivo descrever e analisar a produção científica sobre as Redes de Atenção à Saúde.

\section{Métodos}

Foi realizada uma revisão integrativa da literatura referente à produção do conhecimento sobre a política pública de Redes de Atenção à Saúde. Trabalhou-se com a pesquisa e análise do material científico já produzido de modo sistemático, admitindo-se a elaboração de sínteses a partir dos vários estudos publicados, possibilitando conclusões gerais a respeito de um tema particular em estudo ${ }^{16}$. 
A revisão foi realizada em seis fases distintas: 1) estabelecimento do tema/problema da revisão e elaboração da questão norteadora; 2) seleção dos artigos (estabelecimento de descritores e dos critérios para inclusão/exclusão de publicações); 3 ) categorização dos estudos e definição das informações a serem extraídas dos trabalhos revisados; 4) análise dos estudos selecionados; 5) interpretação dos resultados e discussão; 6) síntese do conhecimento evidenciado nos artigos analisados ${ }^{17}$.

Para elaboração desta revisão integrativa, em primeiro lugar definiu-se a questão central da pesquisa: qual o teor da produção científica publicada em periódicos a respeito da política pública brasileira de Redes de Atenção à Saúde? Para identificar as publicações que compuseram a revisão integrativa desta investigação, realizouse o levantamento em bases de dados da Biblioteca Virtual de Saúde: Literatura Latino-americana e do Caribe em Ciências da Saúde (LILACS), da Scientific Electronic Library Online (SciELO) e do Medical Literature Analysis and Retrieval System Online (MEDLINE), no mês de julho de 2019. Para tanto, foram empregados como descritores a expressão "Rede de Atenção à Saúde" e seus congêneres em inglês "Health Care Network" e espanhol "Red Integrada de Servicios de Salud".

Após a exclusão dos artigos duplicados, procedeu-se à avaliação pela leitura dos títulos e resumos, tendo como critério de inclusão a caracterização enquanto artigo de pesquisa e a abordagem da temática das RAS, não sendo incluídos estudos sobre redes temáticas específicas. Através da leitura integral dos artigos, chegou-se ao corpus da pesquisa, excluindo-se os artigos que não abordavam diretamente a temática das RAS, os que tratavam de experiências de redes em outros países e aqueles que não estavam disponíveis na íntegra.

A fim de sistematizar os dados, foi organizada uma pré-categorização e elaborada uma matriz contendo as seguintes informações: título, ano de publicação, categoria analítica e objetivos do estudo. Foram propostas como categorias analíticas para organização dos resultados: 1) Estudos Conceituais, 2) Relatos de Casos, 3) Análises de Implementação e 4) Estudos de Avaliação, de acordo com o objeto, objetivo ou metodologia de cada estudo revisado.

A análise foi processada pelos autores em reuniões do grupo de pesquisa do projeto "A Rede de Atenção às Urgências e a Produção Viva de Mapas de Cuidados", financiado pelo Conselho Nacional de Desenvolvimento Científico e Tecnológico
(CNPq), que toma as RAS e mais especificamente a RUE como objeto de estudo. Foram formuladas questões-chave, a partir da intensidade de "núcleos de sentido" identificados no corpus da revisão de forma transversal à pré-categorização analítica. Estas questões foram configuradas enquanto categorias temáticas e tiveram o objetivo de orientar a discussão e a elaboração de sínteses.

\section{Resultados}

O estudo possibilitou a análise de 27 artigos científicos publicados em periódicos nacionais e internacionais do campo da Saúde Coletiva dentre os anos de 2010 e 2019 sobre a temática das Redes de Atenção à Saúde. Os resultados parciais de cada etapa do processo de seleção dos artigos estão descritos no fluxo apresentado na Figura 1.

Quanto ao ano de publicação, em 2017 foi observado o maior número de estudos, sete artigos, seguido pelos anos de 2015 e 2018 com seis publicações cada. Nos anos de 2010, 2011, 2013 e 2019 foi encontrado apenas um artigo publicado ao ano. No que concerne ao enfoque das publicações a respeito das RAS, foi proposta uma pré-categorização analítica para organização dos resultados, descrita a seguir, e que é apresentada de forma detalhada nos Quadros de 1 a 4 .

A primeira categoria foi constituída por seis artigos que abordam de forma mais aprofundada o conceito de RAS (Quadro 1). Dentre esses artigos encontramos uma publicação internacional que aborda as "Redes Integradas de Serviços de Saúde" ${ }^{18}$, o conceito precursor das RAS, e também o artigo de Mendes ${ }^{6}$, considerado importante referência teórica para as Redes de Atenção à Saúde no Brasil. Cabe destacar que uma das publicações apresenta o conceito de Redes Vivas ${ }^{19}$, que emerge na produção científica enquanto uma outra racionalidade sobre as redes de saúde.

Já em relação à segunda categoria, Relatos de Casos, foram identificados 8 artigos, descritos no Quadro 2. Vale destacar que apresentam abordagens que abrangem desde vivências locais de equipes de atenção básica, de municípios e regiões de saúde, até um estudo comparativo entre as cidades do Rio de Janeiro e de Lisboa.

$\mathrm{Na}$ terceira categoria encontram-se nove artigos que analisam a implementação de redes (Quadro 3), incluindo seus desafios e estratégias de integração, regionalização, comunicação, apoio institucional e matricial.

A quarta categoria, composta por quatro artigos, trata de estudos de avaliação das RAS (Qua- 


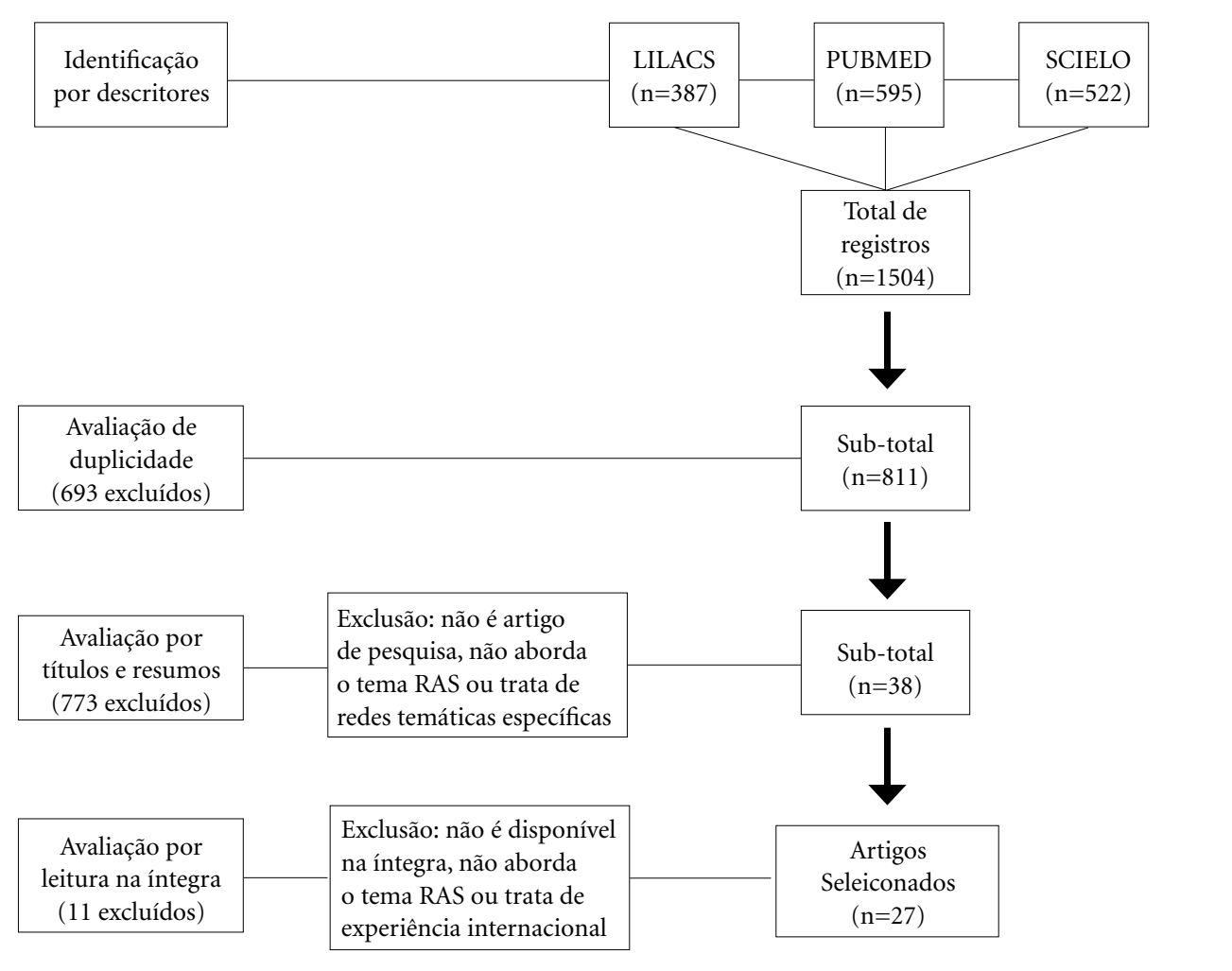

Figura 1. Fluxograma do processo de seleção dos artigos revisados.

Fonte: Elaborado pelos autores.

Quadro 1. Distribuição dos artigos da categoria “Estudos Conceituais", segundo título, ano da publicação e objetivos. LILACS, SciELO, MEDLINE, 2019.

\begin{tabular}{|c|c|c|}
\hline \multicolumn{3}{|r|}{ Categoria - Estudos Conceituais } \\
\hline Título & Ano & Objetivos \\
\hline As Redes de Atenção à Saúde & 2010 & $\begin{array}{l}\text { Apresentar uma resposta social estruturada em sistemas integrados de } \\
\text { saúde: as redes de atenção à saúde }\end{array}$ \\
\hline $\begin{array}{l}\text { Redes integradas de } \\
\text { servicios de salud: hacia La } \\
\text { construcción de un concepto }\end{array}$ & 2012 & $\begin{array}{l}\text { Os autores buscam compreender o conceito de rede e seus elementos } \\
\text { constitutivos, para definir a concepção de Redes Integradas de Serviços } \\
\text { de Saúde }\end{array}$ \\
\hline $\begin{array}{l}\text { Redes Vivas: multiplicidades } \\
\text { girando as existências, sinais } \\
\text { da rua. Implicações para a } \\
\text { produção do cuidado e a } \\
\text { produção do conhecimento } \\
\text { em saúde }\end{array}$ & 2014 & $\begin{array}{l}\text { Sintetizar as experiências recolhidas por um grupo de pesquisadores } \\
\text { vinculados a Micropolítica do Trabalho e o Cuidado em Saúde, da Pós- } \\
\text { Graduação de Clínica Médica } \\
\text { - Universidade Federal do Rio de Janeiro (UFRJ), a partir de suas } \\
\text { investigações sobre a produção do cuidado em saúde, em diferentes } \\
\text { contextos de práticas }\end{array}$ \\
\hline $\begin{array}{l}\text { Perspectivas de região e redes } \\
\text { na política de saúde brasileira }\end{array}$ & 2015 & $\begin{array}{l}\text { Analisar as perspectivas de região e redes adotadas na política de saúde } \\
\text { brasileira, no período de } 2001 \text { a } 2011\end{array}$ \\
\hline $\begin{array}{l}\text { Redes de Atenção à Saúde } \\
\text { sob a luz da Teoria da } \\
\text { Complexidade }\end{array}$ & 2015 & $\begin{array}{l}\text { Refletir acerca dos princípios que norteiam as Redes de Atenção em } \\
\text { Saúde sob a luz dos princípios da Teoria da Complexidade de Edgar } \\
\text { Morin }\end{array}$ \\
\hline $\begin{array}{l}\text { Redes de Atenção à Saúde: } \\
\text { tendências da produção de } \\
\text { conhecimento no Brasil }\end{array}$ & 2019 & $\begin{array}{l}\text { Analisar a tendência de investigação de teses e dissertações brasileiras } \\
\text { da área da saúde sobre o tema Redes de Atenção à Saúde }\end{array}$ \\
\hline
\end{tabular}

Fonte: Elaborado pelos autores. 
Quadro 2. Distribuição dos artigos da categoria "Relatos de Casos”, segundo título, ano da publicação e objetivos. LILACS, SciELO, MEDLINE, 2019.

\begin{tabular}{|c|c|c|}
\hline \multicolumn{3}{|c|}{ Categoria - Relatos de Casos } \\
\hline Título & Ano & Objetivos \\
\hline $\begin{array}{l}\text { A Atenção Básica à Saúde e a } \\
\text { construção das Redes Temáticas de } \\
\text { Saúde: qual pode ser o seu papel? }\end{array}$ & 2012 & $\begin{array}{l}\text { Analisar as reais possibilidades (ou limites ou condicionantes) } \\
\text { para o exercício dessa responsabilidade ampliada posta para a } \\
\text { ABS, examinadas a partir dos achados de uma investigação, em } \\
\text { curso, sobre as múltiplas lógicas do acesso e da utilização de } \\
\text { serviços }\end{array}$ \\
\hline $\begin{array}{l}\text { Atenção Primária à Saúde: } \\
\text { coordenadora do cuidado em redes } \\
\text { regionalizadas? }\end{array}$ & 2016 & $\begin{array}{l}\text { Analisar o alcance da coordenação do cuidado pela Atenção } \\
\text { Primária à Saúde em três regiões de saúde }\end{array}$ \\
\hline $\begin{array}{l}\text { Atenção Primária à Saúde na } \\
\text { coordenação das Redes de Atenção } \\
\text { à Saúde no Rio de Janeiro, Brasil, e } \\
\text { na região de Lisboa, Portugal }\end{array}$ & 2017 & $\begin{array}{l}\text { Analisar comparativamente a organização da APS buscando } \\
\text { identificar os avanços na coordenação das RAS e no manejo das } \\
\text { condições crônicas }\end{array}$ \\
\hline $\begin{array}{l}\text { A implementação de Redes } \\
\text { de Atenção e os desafios da } \\
\text { governança regional em saúde na } \\
\text { Amazônia Legal: uma análise do } \\
\text { projeto Qualisus-Rede }\end{array}$ & 2017 & $\begin{array}{l}\text { Analisar potencialidades, limites e desafios da governança } \\
\text { regional no processo de implementação de redes de atenção à } \\
\text { saúde, em três regiões da Amazônia }\end{array}$ \\
\hline $\begin{array}{l}\text { O trabalho em rede nos casos } \\
\text { de alta Complexidade e de alta } \\
\text { vulnerabilidade: a experiência de } \\
\text { uma UBS }\end{array}$ & 2017 & $\begin{array}{l}\text { Apresentar e analisar os processos de formação de redes de } \\
\text { atenção à saúde em torno de casos complexos e em contextos de } \\
\text { alta vulnerabilidade, atendidos pela Estratégia Saúde da Família } \\
\text { em uma UBS na região da Brasilândia, São Paulo }\end{array}$ \\
\hline $\begin{array}{l}\text { Redes de políticas nas regiões } \\
\text { metropolitanas: o caso do sistema } \\
\text { de saúde brasileiro }\end{array}$ & 2017 & $\begin{array}{l}\text { Discutir os casos das redes de saúde nas regiões metropolitanas } \\
\text { brasileiras, caracterizando seu processo de implementação e } \\
\text { identificando problemas de gestão em vários casos, sendo o caso } \\
\text { da Região Metropolitana de Campinas descrito em mais detalhes }\end{array}$ \\
\hline $\begin{array}{l}\text { Profissionais como produtores } \\
\text { de Redes: tramas e conexões no } \\
\text { cuidado em saúde }\end{array}$ & 2017 & $\begin{array}{l}\text { Analisar, a partir da centralidade do usuário, como os } \\
\text { profissionais de saúde tecem e produzem a rede de cuidados }\end{array}$ \\
\hline $\begin{array}{l}\text { Continuidade do Cuidado em Rede } \\
\text { e os Movimentos de Redes Vivas } \\
\text { nas Trajetórias do Usuário-Guia }\end{array}$ & 2018 & $\begin{array}{l}\text { Descrever e analisar os encontros entre atores da produção } \\
\text { de cuidado, que refletem em movimentos de redes vivas e } \\
\text { potencializam a continuidade do cuidado e a integralidade }\end{array}$ \\
\hline
\end{tabular}

Fonte: Elaborado pelos autores.

dro 4), incluindo a formulação de critérios, as condições sanitárias dos serviços de saúde, a relação entre necessidades e interesses locais e entre a organização necessária e a atual.

\section{Discussão}

A política pública de Redes de Atenção à Saúde tem sido objeto de diversos estudos onde são utilizadas diferentes abordagens metodológicas e concepções. Compreender os conceitos que a embasam, assim como os avanços, limites e desafios do processo de sua implementação, torna-se imperativo enquanto contribuição para o aperfeiçoamento do SUS. Nesta revisão, pode-se observar que o modelo das RAS é formulado a par- tir de um referencial teórico específico, mas não único, havendo influências de outros campos de conceitos e práticas. Enquanto pressupostos do modelo proposto, ficam evidentes a centralidade da atenção básica e a dependência do processo de regionalização da saúde. Enquanto prática, os estudos indicam ações concorrentes ou integradas de dois campos que ora se complementam, ora se contrapõem: a organização de redes para a atenção à saúde, com maior ênfase na estrutura e definição de fluxos, e, o cuidado em saúde como produtor de redes, mais centrado no protagonismo dos usuários e profissionais de saúde.

Para aprofundamento da discussão, utilizamos as seguintes questões-chave: 1) O paradigma das Redes de Atenção à Saúde; 2) A atenção básica como coordenadora das RAS; 3) Regionali- 
Quadro 3. Distribuição dos artigos da categoria “Análises de Implementação", segundo título, ano da publicação e objetivos. LILACS, SciELO, MEDLINE, 2019.

\begin{tabular}{|c|c|c|}
\hline \multicolumn{3}{|c|}{ Categoria - Análises de Implementação } \\
\hline Título & Ano & Objetivos \\
\hline $\begin{array}{l}\text { Organização de redes regionalizadas e } \\
\text { integradas de atenção à saúde: desafios do } \\
\text { Sistema Único de Saúde (Brasil) }\end{array}$ & 2011 & $\begin{array}{l}\text { Analisar os desafios presentes no Sistema Único de } \\
\text { Saúde para promover uma melhor integração entre os } \\
\text { serviços e organizar redes }\end{array}$ \\
\hline $\begin{array}{l}\text { Percepção dos Gestores do Sistema Único } \\
\text { de Saúde acerca dos Desafios da Formação } \\
\text { das Redes de Atenção À Saúde No Brasil }\end{array}$ & 2013 & $\begin{array}{l}\text { Analisar as percepções de gestores sobre os desafios da } \\
\text { formação de Redes de Atenção à Saúde no Brasil }\end{array}$ \\
\hline $\begin{array}{l}\text { Contribuição para a análise da implantação } \\
\text { de Redes de Atenção à Saúde no SUS }\end{array}$ & 2014 & $\begin{array}{l}\text { Analisar a implantação das Redes de Atenção à Saúde } \\
\text { (RAS) com ênfase nas regiões de saúde, ressaltando a } \\
\text { importância de atores locais com adequado perfil de } \\
\text { competência e implicados com a implementação }\end{array}$ \\
\hline $\begin{array}{l}\text { O processo de construção da gestão } \\
\text { regional da saúde no estado de São Paulo: } \\
\text { subsídios para a análise }\end{array}$ & 2015 & $\begin{array}{l}\text { Com o intuito de discutir o processo de implementação } \\
\text { do COAP em algumas regiões paulistas, o presente } \\
\text { artigo fornece subsídios para a compreensão do } \\
\text { processo de pactuação regional da saúde no Estado, } \\
\text { verificando suas potencialidades e limites }\end{array}$ \\
\hline $\begin{array}{l}\text { Uma Noção de Matriciamento que } \\
\text { Merece Ser Resgatada para o Encontro } \\
\text { Colaborativo entre Equipes de Saúde e } \\
\text { Serviços no SUS }\end{array}$ & 2015 & $\begin{array}{l}\text { Revisitar o conceito original de matriciamento e } \\
\text { analisá-lo à luz da teoria matemática do cálculo } \\
\text { matricial e das normativas federais }\end{array}$ \\
\hline $\begin{array}{l}\text { Reflexões para Construção de uma } \\
\text { Regionalização Viva }\end{array}$ & 2017 & $\begin{array}{l}\text { Analisar o complexo processo da regionalização da } \\
\text { saúde e os desafios decorrentes de sua implementação }\end{array}$ \\
\hline Regionalização e Redes de Saúde & 2018 & $\begin{array}{l}\text { Refletir sobre os desafios da construção das Regiões e } \\
\text { Redes de Saúde no Brasil }\end{array}$ \\
\hline $\begin{array}{l}\text { Apoio Institucional na ótica de gestores, } \\
\text { apoiadores e trabalhadores: uma } \\
\text { aproximação da realidade a partir de } \\
\text { diferentes lugares }\end{array}$ & 2018 & $\begin{array}{l}\text { Analisar os sentidos e significados que as equipes de } \\
\text { Saúde da Família (EqSF), os gestores e os apoiadores } \\
\text { atribuem ao processo de trabalho do Apoio } \\
\text { Institucional municipal em dois municípios da Bahia }\end{array}$ \\
\hline $\begin{array}{l}\text { Estratégias de Comunicação das Secretarias } \\
\text { Municipais de Saúde: Desafios para as } \\
\text { Redes de Atenção à Saúde }\end{array}$ & 2018 & $\begin{array}{l}\text { Descrever as estratégias de comunicação em saúde de } \\
19 \text { municípios paranaenses }\end{array}$ \\
\hline
\end{tabular}

Fonte: Elaborado pelos autores.

zação, redes e governança regional; 4) O cuidado em rede; e 5) Desafios para implementação das RAS.

\section{O paradigma das Redes de Atenção à Saúde}

O modelo conceitual das RAS foi proposto como paradigma para organização do SUS no Brasil na última década e adotado tanto pelo Ministério da Saúde como por gestores estaduais e municipais de saúde como política pública ${ }^{13}$. Publicação de Mendes ${ }^{9}$, autor que é importante referência para o tema, faz críticas aos sistemas hierarquizados, fragmentados, orientados para assistência às condições agudas, e define as RAS como organizações poliárquicas de conjuntos de serviços de saúde, vinculados entre si por uma missão única, por objetivos comuns e por uma ação cooperativa e interdependente, que permitem ofertar uma atenção contínua e integral a determinada população, coordenada pela atenção primária à saúde - prestada no tempo certo, no lugar certo, com o custo certo, com a qualidade certa e de forma humanizada, e com responsabilidades sanitárias e econômicas por esta população. Esse conceito foi balizador para a RAS e as redes temáticas que foram subsequentemente pactuadas, no plano discursivo e normativo, entre os gestores do SUS no âmbito da Comissão Intergestores Tripartite e posteriormente consubstanciadas nas respectivas portarias ${ }^{14}$. Arruda et al. ${ }^{20}$, ao analisarem o conceito de RAS sob a luz da Teoria da Complexidade, identificam como suas características principais: a formação de relações 
Quadro 4. Distribuição dos artigos da categoria “Estudos de Avaliação", segundo título, ano da publicação e objetivos. LILACS, SciELO, MEDLINE, 2019.

\begin{tabular}{|l|c|l|l|}
\hline \multicolumn{1}{|c|}{ Título } & Ano & \multicolumn{1}{|c|}{ Objetivos } \\
\hline $\begin{array}{l}\text { Redes de Atenção à Saúde do } \\
\text { SUS: } 25 \text { anos de uma contradição } \\
\text { fundamental entre a Organização } \\
\text { Necessária e a Organização Atual }\end{array}$ & 2014 & $\begin{array}{l}\text { Examinar a contradição entre a organização necessária } \\
\text { das Redes de Atenção à Saúde do SUS, de forma integral e } \\
\text { integrada em todo o território nacional, e a essência federativa } \\
\text { e municipalista da organização atual do SUS, estruturalmente } \\
\text { fragmentada e desintegrada }\end{array}$ \\
\hline $\begin{array}{l}\text { Integração assistencial em região de } \\
\text { saúde: paradoxo entre necessidades } \\
\text { regionais e interesses locais }\end{array}$ & 2016 & $\begin{array}{l}\text { Identificar e analisar o desenvolvimento de estratégias } \\
\text { regionais para a integração assistencial no contexto das regiões } \\
\text { de saúde, com especial foco no papel da Atenção Primária à } \\
\text { Saúde (APS) }\end{array}$ \\
\hline $\begin{array}{l}\text { Rede de atenção e condições } \\
\text { sanitárias das unidades de saúde da } \\
\text { família: há alguma relação? }\end{array}$ & 2016 & $\begin{array}{l}\text { Discutir as concepções e modos/estratégias de } \\
\text { operacionalização da rede de atenção à saúde, bem como as } \\
\text { condições sanitárias de USF }\end{array}$ \\
\hline $\begin{array}{l}\text { Coordenação do cuidado e ordenação } \\
\text { nas redes de atenção pela Atenção } \\
\text { Primária à Saúde uma proposta de } \\
\text { itens para avaliação destes atributos }\end{array}$ & 2017 & $\begin{array}{l}\text { Propor um conjunto de elementos-chave que permitam avaliar } \\
\text { a coordenação do cuidado individual e o papel de ordenação } \\
\text { das redes de atenção à saúde pela APS para no futuro compor } \\
\text { um instrumento de avaliação das redes }\end{array}$ \\
\hline
\end{tabular}

Fonte: Elaborado pelos autores.

horizontais entre os pontos de atenção, tendo o centro de comunicação na atenção primária; a centralidade das necessidades em saúde de uma população; a responsabilização pela atenção contínua e integral; o cuidado multiprofissional; e, o compartilhamento de objetivos e compromissos com os resultados sanitários e econômicos.

A formulação da política de Redes de Atenção à Saúde no Brasil tem importante influência das Redes Integradas de Serviços de Saúde, preconizadas pela OPAS, que pressupõem a atenção a uma população determinada, serviços ou estabelecimento de saúde com diferentes níveis de atenção e complexidade e uma definição territorial ${ }^{5}$. O pressuposto central em tal definição é que a aplicação do conceito de redes contribui para a articulação, interdependência e coordenação de atores públicos, privados e mistos, com a incorporação de tecnologias especializadas ampliando o acesso, melhorando também a conectividade na rede de saúde e aumentando sua governança ${ }^{18}$. Ainda que no plano discursivo tome as regiões de saúde como espaço de planejamento das RAS na perspectiva de produção da integralidade do cuidado e da articulação do conjunto de políticas públicas intersetoriais necessárias para tanto, questiona-se o quanto tal conceito de redes é capaz de considerar, por um lado, as grandes diferenças regionais, a estrutura econômica-social heterogênea, as mudanças demográficas, nutri- cionais e epidemiológicas de um país superpopuloso e de dimensões continentais como o Brasil, e por outro, a dinâmica micropolítica envolvida na produção do cuidado, que parece escapar dos postulados que embasam a formulação do conceito de redes ${ }^{2}$.

Nesse sentido, para além do paradigma das RAS, identifica-se a formulação recente de outro modelo conceitual, o das Redes Vivas, definidas por Merhy et al. ${ }^{19}$ como modo de produção das conexões existenciais de indivíduos e coletivos, em diferentes contextos de grupalidade e modos de viver, socialmente. Em um estudo com usuários-guia, Hadad e Jorge ${ }^{21}$ identificaram redes vivas, produzidas em ato, no encontro entre profissionais e usuários a partir das necessidades em saúde, mas também a falta de rede, que impactou em barreiras dificultadoras de acesso.Também Maximino et al. ${ }^{22}$ observaram redes de atenção amplas, operacionalizadas de diversas maneiras e compostas por múltiplos vetores e elementos formais e informais, visíveis e invisíveis, objetivos e subjetivos que contribuem para facilitar ou dificultar a construção de pontos de articulação entre serviços, pessoas e recursos para o cuidado. Para Merhy et al. ${ }^{19}$ as Redes Vivas são fragmentárias e em acontecimento, hipertextuais, ou seja, às vezes são circunstanciais, montam e desmontam, e às vezes elas se tornam mais estáveis, mas comportam-se mais como lógicas de redes digi- 
tais, que podem emergir em qualquer ponto sem ter que obedecer a um ordenamento lógico das redes analógicas, como um hipertexto.

Assim, percebe-se diferentes concepções de redes de saúde que coexistem e se interconectam, evidenciando a necessidade de estudos para análise e aprofundamento da relação entre estrutura e ação, norma e produção singular, a partir dos modelos conceituais de "Não Rede" (a fragmentação), "Redes de Atenção à Saúde" (organização sistêmica) e "Rede Vivas" (produção singular), suas possibilidades e limites na modelagem dos sistemas de saúde e na produção do modo de cuidar.

\section{A atenção básica como coordenadora das RAS}

A coordenação das RAS pela atenção básica emerge como condição estruturante do modelo. Para Mendes ${ }^{9}$, o centro de comunicação das redes de atenção à saúde é o nó intercambiador no qual se coordenam os fluxos e contrafluxos do sistema de atenção à saúde e é constituído pela atenção primária à saúde.

Chueiria et $a l .{ }^{23}$ formularam uma proposta de itens para avaliação da coordenação das RAS pela atenção primária, composta pelos seguintes atributos: 1) atenção primária que cobre toda a população; 2) está organizada em equipes multiprofissionais; 3 ) é a principal porta de entrada do sistema; 4) é responsável pela coordenação do cuidado; 5) é resolutiva, sendo capaz de resolver os principais problemas de saúde de sua população. Em pesquisa desenvolvida por Peiter et al. ${ }^{15}$, em que analisaram as tendências da produção científica em dissertações e teses de pós-graduação no Brasil sobre as RAS, a Atenção Primária à Saúde (APS) como ordenadora da rede apresentou-se com a maior frequiência de estudos dentre as categorias estudadas.

Lapão et al..$^{24}$, em estudo comparativo, observaram que questões históricas, culturais, políticas e jurídicas determinam diferenças na Atenção Primária à Saúde (APS) como coordenadora das RAS no Rio de Janeiro e em Lisboa: em Lisboa utiliza-se o modelo de APS abrangente que possui maturidade suficiente na coordenação do seu sistema, enquanto o Rio de Janeiro sofre resquícios históricos de uma APS seletiva. No Brasil, apesar da percepção dos gestores de que as RAS devem ser alicerçadas na valorização e no reconhecimento da atenção básica como instância responsável pelo ordenamento do sistema e pela coordenação do cuidado ${ }^{25}$, estes atributos ainda permanecem como um dos principais desafios para a organização de redes regionalizadas e integradas no SUS ${ }^{26}$. Pouco pautada nas reuniões das Comissões Intergestores Regionais (CIR), a meta de conformação de Redes de Atenção à Saúde, coordenadas pela atenção básica, ainda é um objetivo distante diante da premência de garantir acesso à atenção especializada e de urgência/ emergência hospitalar na região de saúde, tema que dominou as discussões das CIR, ficando a atenção básica circunscrita aos debates municipais ${ }^{27}$.

Cecílio et al. ${ }^{28}$ descrevem os seguintes limites para a atenção básica assumir o papel de coordenadora do cuidado: a rede básica é vista como lugar de coisas simples, há uma impotência compartilhada entre usuários e equipes quando se trata da rede básica funcionar como coordenadora do cuidado, indicando como ela não reúne condições materiais (tecnológicas, operacionais, organizacionais) e simbólicas (valores, significados e representações) de deter a posição central da coordenação das redes temáticas de saúde. Também as condições de infraestrutura das Unidades Básicas de Saúde, ou suas condições sanitárias, podem interferir na organização e na oferta de serviços prestados, na ineficiência da comunicação entre os níveis de atenção e em falhas no processo de regulação, gerando, portanto, problemas para a operacionalização da RAS ${ }^{29}$. Além disso, na função de porta de entrada preferencial do sistema, a APS se depara com forte concorrência de serviços ambulatoriais hospitalares e de pronto atendimento, desarticulados da rede ${ }^{30}$.

Assim, observa-se que a coordenação pela atenção básica é ao mesmo tempo uma premissa e um desafio para as RAS, conforme a produção científica evidenciada nesta revisão.

\section{Regionalização, Redes e Governança Regional}

A relação entre os processos de regionalização, governança regional e produção das RAS tem sido reconhecida enquanto interdependente e é objeto de estudo no Brasil. Para Mendes ${ }^{9}$ a governança das redes de atenção à saúde, no SUS, deve ser feita por meio de arranjos interfederativos, coerentes com o federalismo cooperativo que se pratica no Brasil. São as comissões intergestores que se materializam: no plano nacional, na Comissão Intergestores Tripartite (CIT); nos estados, nas Comissões Intergestores Bipartite (CIB); e nas regiões de saúde, nas Comissões Intergestores Regionais (CIR). 
Albuquerque e Viana ${ }^{31}$ identificaram três gionalização e organização de redes no Brasil, orientadas por diferentes concepções teóricas e políticas: a) região normativa com redes regionalizadas e hierarquizadas (Norma Operacional de Assistência à Saúde - NOAS 2001 e 2002); região negociada com redes integradas e regionalizadas (Pacto pela Saúde - Portaria 399/2006); e região negociada e contratualizada com redes de atenção à saúde (Portaria 4.279/2010 e Decreto 7.508/2011). Assim, fica evidenciada a recente transição das regiões normativas para as regiões negociadas e das redes hierarquizadas para as redes integradas, regionalizadas e contratualizadas.

A organização de redes regionalizadas no SUS depende de aperfeiçoamento na gestão interfederativa nas regiões de saúde para qualificar a pactuação de responsabilidades entre as esferas de governo ${ }^{32}$. Desta forma, o fortalecimento da governança e do planejamento regional está sujeito a uma compreensão mais aprofundada do contexto: identificação das causas dos problemas, da dinâmica e da estrutura de poder das organizações, dos determinantes do comportamento dos usuários, assim como atores locais conhecedores e capacitados para implementação das RAS $^{32}$. Para Shimizu ${ }^{25}$, que pesquisou a percepção dos gestores sobre os desafios para a formação das RAS no Brasil, a operacionalização deste processo não pode seguir modelos prescritivos, $\mathrm{e}$ requer investimentos que incluam o aprimoramento das dimensões técnicas e políticas.

$\mathrm{Na}$ dimensão técnica, aponta para o aperfeiçoamento de estratégias de contratualização para a garantia da provisão de ações e serviços de saúde de diferentes densidades tecnológicas, bem como à logística para orientar o usuário no itinerário das malhas da rede, sistema de regulação, com normas e protocolos claros para orientar o acesso à rede de serviços, definir competências e responsabilidades ${ }^{25}$. Para Leite ${ }^{33}$, as redes se implementam por meio de Linhas de Cuidados, em pactuações entre os diferentes atores para a construção de um planejamento estratégico e de investimento, incluindo mudanças estruturais, planos de Educação Permanente e qualificação dos processos de regulação. Gomes ${ }^{34}$, em oposição ao processo de descentralização em curso, chega a propor a criação em nível federal e atuação nacional de um novo ente jurídico exclusivamente voltado para o SUS, que seja o mais convergente possível para a organização necessária das redes, superando a autonomia político-administrativa dos gestores loco-regionais.
Já na dimensão política, há disputas de poder e conflitos no âmbito regional, inclusive as formatadas pelo sistema de serviços de saúde privado que requerem fortes mecanismos de regulação $0^{25}$. Para Mendes et al. ${ }^{35}$, a regionalização, mais do que um processo de organização das ações e dos serviços de saúde no território, visando assegurar a integralidade da atenção, é uma construção política que deve favorecer o diálogo entre os atores locais e os gestores federados para o reconhecimento e enfrentamento das necessidades de saúde dos territórios específicos.

Estudo realizado em regiões de saúde da Amazônia, a partir da implementação do projeto QUALISUS-Rede, observou que houve fortalecimento da governança e a integração de modo diferenciado entre as regiões, a depender da capacidade de direção e consenso entre os atores, dificultando a superação das desigualdades regionais ${ }^{36}$. Chioro dos Reis et al. ${ }^{2}$, ao proporem a construção de uma Regionalização Viva, indicam que esta requer um forte investimento dos gestores para que o espaço regional possa se tornar um espaço vivo e potente de gestão compartilhada. Sem essa aposta será reduzido a um espaço formal, instituído pelas normas e sem potência para implementar um SUS de qualidade em cada região, capaz de produzir mais vida para todos os brasileiros.

Portanto, percebe-se que um processo intenso de cogestão e planejamento regional é imprescindível para a implementação das RAS, constituindo-se a governança regional também como um desafio, devendo ser observados seus aspectos técnicos e políticos, além do contexto federativo brasileiro.

\section{O Cuidado em Rede}

As redes conformadas no processo de cuidado em saúde valorizam o encontro profissionalusuário como produtor de tramas e conexões, pois provocam a construção das redes em maior ou menor potência. Esses momentos impactam diretamente no desfecho de cuidado dos casos $^{21}$. Cecílio et al. ${ }^{28}$, em pesquisa sobre o papel da atenção básica nas redes temáticas, refletem na conclusão do artigo sobre a falsa imagem de harmonia entre os cuidados e de uma complexa trama traçada pelos indivíduos na composição singular da sua rede de cuidados.

Para Maximino et al. ${ }^{22}$ as redes formais coexistem com as informais, algumas vezes não reconhecidas pelos profissionais, apontando a necessidade de se valorizar o usuário e suas redes como 
protagonistas na busca de apoio. Segundo Merhy et al. ${ }^{19}$, os usuários são Redes Vivas de si próprios; estão o tempo inteiro produzindo movimentos, elaborando saberes, construindo e partilhando cuidados. Quem pede as redes, na maioria das vezes, são os usuários e a rede não está já dada feito um arcabouço a ser preenchido de forma protocolar, pois vão em acontecimentos sendo tecidas. Já para Arruda et al. ${ }^{20}$, redes tecidas pelos usuários são compreendidas como uma situação indesejável e disfuncional: como uma orquestra em que cada instrumento toca uma música diferente, a desarmonia se instala, os usuários entram no sistema por todas as portas e forma-se um labirinto com caminhos diferentes a serem tomados e, que, muitas vezes, não são compreendidos por eles e nem mesmo pelos profissionais que fazem parte desse complexo processo.

A necessidade de investimento para formação dos trabalhadores para práticas integrais em saúde que considerem o usuário como protagonista do seu cuidado é destacada por Hadad e Jorge ${ }^{21}$. Para Nobre et al. ${ }^{37}$ a formação de rede aproxima os profissionais do território, explora a potencialidade de assistência e possibilita uma abordagem mais integrada, proporcionando um atendimento mais humanizado. Além da Educação Permanente em Saúde, outros arranjos de gestão podem induzir estas práticas. O Apoio Institucional se apresenta como uma possibilidade de cogestão, numa tentativa de diminuir os ruídos, conhecer os limites, respeitar o lugar do outro, compreender a si, compreender os outros e construir um ambiente de trabalho e de cuidado de forma mais saudável e prazerosa ${ }^{38}$. Segundo Medeiros $^{39}$, o Apoio Matricial pode contribuir para a integralidade do cuidado e deve-se rejeitar a regra produtora de modelos e posições cristalizadas em detrimento da lei libertadora dos elementos e sistemas equacionados na equipe matricial: lei que não pode ser inscrita em um manual, pois deve se escrever em cada caso em que o matriciamento seja convocado, para que cumpra o papel originalmente intuído.

Assim, observa-se a dimensão micropolítica do trabalho e do cuidado em saúde como uma possibilidade para as redes, uma outra racionalidade a ser considerada, em que o protagonismo do usuário e a autonomia dos profissionais de saúde podem produzir redes próprias e mapas de cuidados singulares a partir das necessidades em saúde.

\section{Desafios para a Implementação das Redes de Atenção à Saúde}

Os desafios para a implementação das RAS foi objeto de estudo de diversas pesquisas na literatura revisada, evidenciando-se diversas ordens de desafios, incluindo estruturais, funcionais e políticos.

Uma análise dos sistemas de atenção à saúde, feita numa perspectiva internacional, mostra que eles são fragmentados, voltados para atenção às condições agudas e às agudizações de condições crônicas ${ }^{9}$. No Brasil, a capacidade de coordenação das redes mostra-se afetada pela fragmentação ${ }^{27}$ e dificulta a promoção pelo SUS da saúde como um direito social ${ }^{25}$. Segundo Arruda et al..$^{20}$ as RAS, teoricamente, organizam-se em graus crescentes de complexidade, onde a população deveria usufruir dos vários níveis por meio de fluxos coerentes. Porém, na prática, esse fluxo esbarra em um funcionamento truncado, burocrático e desarticulado que não considera as necessidades e os movimentos reais das pessoas dentro do sistema, o que o torna lento e em muitos casos com resultados insatisfatórios.

A insuficiência de recursos decorrentes do baixo investimento público em saúde é um problema estrutural para o SUS e dificulta o planejamento regional para a organização das RAS $^{20,26,33,35}$. Para Chioro dos Reis et al. ${ }^{2}$ o comprometimento do orçamento municipal com saúde para muito além do mínimo obrigatório (15\%) é pernicioso para a gestão equilibrada das cidades. Continua-se observando baixa participação dos Estados no financiamento e os recursos estaduais em muitos cenários não têm sido pactuados com os gestores municipais. Iniciativas recentes congelaram os gastos públicos federais por duas décadas, apontando para a inviabilidade definitiva do SUS. Sem a perspectiva de novos recursos, torna-se inviável o planejamento e a programação regional.

Outro desafio estrutural no Brasil é a política de formação de profissionais de saúde. Para Arruda et al. ${ }^{20}$ há uma política insuficiente de formação de pessoal para o SUS, incluindo aspectos quantitativos e qualitativos. Há necessidade de processos de formação e educação, com consequente disponibilização de profissionais para o sistema público ${ }^{26}$ e a necessidade de engajar os trabalhadores em saúde e, especificamente, os médicos, como agentes de mudança para a constituição das regiões e redes de saúde ${ }^{40}$.

Dentre os desafios de funcionamento das RAS, observa-se a necessidade de superação das 
limitações de algumas estratégias. Para Leite et al. ${ }^{33}$ há necessidade de melhoria nos sistemas de informação e falhas nos processos de monitoramento e avaliação das redes. Já Velho et al. ${ }^{41}$ destacam as fragilidades dos processos de comunicação: as redes estão alicerçadas de forma frágil e apesar dos programas de promoção e campanhas, ainda não há um processo que empreenda medidas capazes de levar ao cidadão informação para que ele assuma a corresponsabilidade na construção e condução do SUS. Para Chioro dos Reis et al. ${ }^{2}$ os principais desafios são o planejamento e dimensionamento da rede de serviços, a produção de redes vivas de cuidados, a regulação e a capacidade de coordenação do sistema regional. Viana et al. ${ }^{40}$ indicam a necessidade de uma liderança institucional a ser exercida pelos estados da federação. Para Mendes et al..$^{35}$ a esfera estadual de poder ainda apresenta dificuldades para assumir a efetiva coordenação desse processo, restringindo-se ao papel de gestor da prestação de seus próprios serviços.

$\mathrm{Na}$ dimensão política, os principais desafios estão nas relações entre os diversos atores que compõem os territórios onde se constroem as RAS. Para Albuquerque e Viana ${ }^{31}$ os modelos de organização territorial do sistema de saúde e suas combinações, em constante disputa no âmbito da federação, são condicionados por dinâmicas espaciais e relações entre Estado, mercado e sociedade na condução da política e das redes de saúde. Também os ciclos eleitorais, as relações interfederativas e as pactuações regionais são processos políticos com impacto nas RAS 2 . Mendes et al. $^{35}$ destacam a necessidade das pactuações contemplarem solidariamente os municípios com maiores dificuldades socioeconômicas, epidemiológicas, demográficas e de oferta de serviços, com o intuito de fortalecer o conjunto de gestores municipais e possibilitar o maior enfrentamento dos desafios regionais; e, além disso, ressaltam que as fragilidades da construção político-administrativa dos Colegiados de Gestão Regional os tornam mais vulneráveis aos interesses particulares em detrimento dos coletivos.

\section{Considerações finais}

A produção científica sobre as RAS é densa e inclui pesquisas conceituais, estudos de casos, análises de estratégias de implementação, abarcam propostas de métodos de avaliação, além de discutirem a produção do cuidado em rede. Há ainda investigações sobre os desafios para a implementação das RAS, abordando diferentes concepções e recortes temáticos.

O modelo conceitual das Redes de Atenção à Saúde é apresentado enquanto um novo paradigma de organização para o SUS e busca reorientar o sistema para garantir a integralidade e melhores resultados sanitários. Fica evidente como o modelo conceitual impacta na perspectiva de conformação das redes. Neste modelo, a atenção básica assume um papel central enquanto coordenadora do cuidado e ordenadora do sistema, o que é ao mesmo tempo uma premissa e um desafio. Há aqui um questionamento de quanto esta premissa tem ajudado na conformação real das redes. Também a regionalização e a governança regional trazem a relação interfederativa como aposta e limitação, devendo ser observados seus aspectos técnicos, políticos e a forma como são produzidas, inclusive pelas particularidades da estrutura federativa no contexto brasileiro. Por outro lado, identifica-se uma outra produção conceitual e de práticas, as Redes Vivas, em que a dimensão micropolítica do trabalho e do cuidado em saúde se apresentam como uma possibilidade para as redes. Trata-se de outra racionalidade, ao apresentar uma concepção de rede menos funcionalista. Tal concepção traz para o cenárioo protagonismo do usuário e a relação deste com o trabalhador de saúde, também dotado de autonomia, que podem produzir redes próprias, a partir das necessidades em saúde, conformando redes rizomáticas.

Além dos desafios da governança regional, do subfinanciamento, transformado agora em desfinanciamento do SUS, dos poderes instituídos, do modelo assistencial hegemônico e das potências e limitações da atenção básica, a modelagem organizativa do SUS transita entre um sistema fragmentado (o caos), as redes poliárquicas (a organização) e as tramas e conexões produzidas no espaço do cuidado (a criatividade). As diferenças entre as várias possibilidades aqui apresentadas não nos parece uma mera questão conceitual ou de escolha fortuita de cada ator. São indicativas de diferentes caminhos.

Algumas limitações precisam ser mencionadas ao final desta pesquisa. Primeiramente, grande parte dos estudos revisados foi baseada em métodos majoritariamente qualitativos que, embora tragam percepções, conceitos e elaborações teórico-práticas importantes, expõem pouca produção de evidências através de dados numéricos e indicadores de saúde. Em outra direção, o foco na pesquisa de uma política pública de saúde brasileira, mesmo que influenciada por experiên- 
cias e modelos internacionais, estabelece desafios no que se refere à comparação entre a realidade nacional e as experiências de outros países. Consequentemente, os resultados revisados devem ser interpretados com cautela, considerando-se as limitações de generalização e de síntese.

Por fim, esta revisão integrativa aponta para duas questões centrais e não para uma conclusão.

A primeira delas é da ordem de um problema teórico-prático, pois fica a pergunta: como superar a fragmentação do cuidado, garantindo a integralidade, compondo as redes de atenção à saúde, a partir de concepções teóricas distintas? Ou seja, como produzir um SUS de forma não caótica, que ao estruturar as redes formais, necessárias à própria gestão do sistema e dos serviços, inclua a produção viva do cuidado em saúde, a partir das práticas criativas realizadas cotidianamente diante dos múltiplos e singulares encontros dos usuários com os trabalhadores de saúde?

A segunda questão que se coloca, de ordem sistêmica e política, é: como a produção das redes de cuidado pode impactar as relações interfederativas, o processo de financiamento do SUS, a conformação dos vários tipos de serviços de saúde, o acesso da população ao sistema e centralmente a qualidade e potência dos processos de cuidado em saúde?

\section{Colaboradores}

LFN Tofani: colaborou na elaboração e supervisão do projeto, na coleta e análise dos dados, na elaboração, redação e revisão final do artigo. A Chioro: colaborou na elaboração e supervisão do projeto, na análise dos dados, na elaboração e revisão final do artigo. LAC Furtado: colaborou na elaboração e supervisão do projeto, na análise dos dados, na elaboração e revisão crítica do artigo. $\mathrm{R}$ Andreazza: colaborou na elaboração e supervisão do projeto, na análise dos dados, na elaboração e revisão crítica do artigo. CF Guimarães: colaborou na elaboração e supervisão do projeto e na análise dos dados. DGCF Feliciano: colaborou na coleta, análise dos dados e revisão do artigo. GR Silva: colaborou na coleta, análise dos dados e revisão do artigo. LM Bragagnolo: colaborou na análise dos dados e revisão do artigo. 


\section{Referências}

1. Viana ALA, Machado CV. Descentralização e coordenação federativa: a experiência brasileira na saúde. Cien Saude Colet 2009; 14(3):807-817.

2. Chioro dos Reis AA, Sóter APM, Furtado LAC, Pereira SSS. Reflexões para a Construção de uma Regionalização Viva. Cien Saude Colet 2017; 22(4):1045-1054.

3. Organización Panamericana de La Salud (OPAS). Redes integradas de servicios de salud: conceptos, opciones de politica e hoja de ruta para sua implementación em las Américas. Washington, D.C.: OPAS; 2010.

4. Mendes EV. As Redes de Atenção à Saúde. Brasília: OPAS; 2011.

5. Organização Pan-Americana de Saúde (OPAS). Organização Mundial de Saúde (OMS). A atenção à saúde coordenada pela APS: construindo as redes de atenção no SUS. Brasília: OPAS, OMS; 2010.

6. Tabarquino Muñoz R, Pulgarin Henao D, Giraldo Varón A. Dinámica de una red integral de prestadores de servicios de salud (RIPSS). Gerencia Polit Salud 2018; 17(34):1-12.

7. Almeida PF, Oliveira SC, Giovanella L. Integração de rede e coordenação do cuidado: o caso do sistema de saúde do Chile. Cien Saude Colet 2018; 23(7):22132228.

8. Giovanella L. Redes integradas, programas de gestão clínica e generalista coordenador: análise das reformas recentes do setor ambulatorial na Alemanha. Cien Saude Colet 2011; 16(Supl. 1):1081-1096.

9. Mendes EV. As Redes de Atenção à Saúde. Cien Saude Colet 2010; 15(5):2297-2305.

10. Silva SF, Magalhães Junior HM. Redes de atenção à saúde: conceito, modelo e diretrizes. In: Redes de Atenção à Saúde no SUS. Campinas: IDISA: CONASEMS; 2008.

11. Cecílio LCO. Modelos Tecno-assistenciais em saúde: da pirâmide ao círculo, uma possibilidade a ser explorada. Cad Saude Publica 1997; 13(3):469-478.

12. Hartz ZMA, Contandriopoulos AP. Integralidade da atenção e integração de serviços de saúde: desafios para avaliar a implantação de um "sistema sem muros”. Cad Saude Publica 2004; 20(2):331-336.

13. Brasil. Ministério da Saúde (MS). Portaria no 4.279, de 30 de dezembro de 2010. Estabelece diretrizes para a organização da Rede de Atenção à Saúde no âmbito do Sistema Único de Saúde (SUS). Diário Oficial da União 2010; dez 31.

14. Brasil. Ministério da Saúde (MS). Secretaria de Atenção á Saúde. Implantação das Redes de Atenção à Saúde e outras estratégias da SAS. Brasília: MS; 2014

15. Peiter CC, Santos JLG, Lanzoni GMM, Mello ALSF Costa MFBNA, Andrade SR. Redes de atenção à saúde: tendências da produção de conhecimento no Brasil. Esc Anna Nery Rev Enferm 2019; 23(1):e20180214.

16. Moreira MADM, Lustosa AMDF, Barros EO, Batista JBV, Duarte MCS. Políticas públicas de humanização: revisão integrativa da literatura. Cien Saude Colet 2015; 20(10):3231-3242.

17. Mendes KDS, Silveira RCCP, Galvão CM. Revisão integrativa: método de pesquisa para a incorporação de evidências na saúde e na enfermagem. Texto Contexto Enferm 2008; 17(4):758-764.
18. Barragán Bechara JC, Riaño Casallas MI, Martínez M. Redes integradas de servicios de salud: hacia La construcción de un concepto. Universidad Salud 2012; 14(2):186-196.

19. Merhy EE, Gomes MPC, Silva E, Santos MFL, Cruz KT, Franco TB. Redes Vivas: multiplicidades girando as existências, sinais da rua. Implicações para a produção do cuidado e a produção do conhecimento em saúde. Divulg Saude Debate 2014; 52:153-164.

20. Arruda C, Lopes SGR, Koerich MHAL, Winck DR, Meirelles BHS, Mello ALSF. Redes de atenção à saúde sob a luz da teoria da complexidade. Esc Anna Nery Rev Enferm 2015; 19(1):169-173.

21. Hadad ACAC, Jorge AO. Continuidade do cuidado em rede e os movimentos de redes vivas nas trajetórias do usuário-guia. Saude Debate 2018; 42(n. esp. 4):198-210

22. Maximino VS, Liberman F, Frutuoso MF, Mendes R. Profissionais como produtores de redes: tramas e conexões no cuidado em saúde. Saude Soc 2017; 26(2):435-447.

23. Chueiri PS, Harzheima E, Takeda SMP. Coordenação do cuidado e ordenação nas redes de atenção pela Atenção Primária à Saúde uma proposta de itens para avaliação destes atributos. Rev Bras Med Fam Comunidade 2017; 12(39):1-18.

24. Lapão LV, Arcêncio RA, Popolin MP, Rodrigues LBB. Atenção Primária à Saúde na coordenação das Redes de Atenção à Saúde no Rio de Janeiro, Brasil, e na região de Lisboa, Portugal. Cien Saude Colet 2017; 22(3):713-724.

25. Shimizu HE. Percepção dos gestores do Sistema Único de Saúde acerca dos desafios da formação das Redes de Atenção à Saúde no Brasil. Physis 2013; 23(4):11011122.

26. Silva SF. Organização de redes regionalizadas e integradas de atenção à saúde: desafios do Sistema Único de Saúde (Brasil). Cien Saude Colet 2011; 16(6):27532762.

27. Almeida PF, Santos AM, Santos VP, Silveira Filho RM Integração assistencial em região de saúde: paradoxo entre necessidades regionais e interesses locais. Saude Soc 2016; 25(2):320-335

28. Cecilio LCO, Andreazza R, Carapinheiro G, Araújo EC, Oliveira LA, Andrade MGG, Meneses CS, Pinto NRS, Reis DO, Santiago S, Souza ALM, Spedo SM. A Atenção Básica à Saúde e a construção das redes temáticas de saúde: qual pode ser o seu papel? Cien Saude Colet 2012; 17(11):2893-2902.

29. Barreto LA, Souza MKB, Silva EAL. Rede de atenção e condições sanitárias das unidades de saúde da família: há alguma relação? Rev Enferm UERJ 2016; 24(6):e17440.

30. Almeida PF, Santos AM. Atenção Primária à Saúde: coordenação do cuidado em redes regionalizadas? Rev Saude Publica 2016; 50:80.

31. Albuquerque MV, Viana ALD. Perspectivas de região e redes na política de saúde brasileira. Saude Debate 2015; 39(n. esp.):28-38.

32. Silva SF. Contribuição para a análise da implantação de Redes de Atenção à Saúde no SUS. Divulg Saude Debate 2014; 52:165-176. 
33. Leite JPA, Spatti AC, Campos ML. Redes de Políticas nas Regiões Metropolitanas: O Caso do Sistema de Saúde Brasileiro. Physis 2017; 27(4):1039-1064.

34. Gomes RM. Redes de Atenção à Saúde do SUS: 25 anos de uma contradição fundamental entre a Organização Necessária e a Organização Atual. Saude Debate 2014; 38(103):938-952.

35. Mendes A, Louvison MCP, Ianni AMZ, Leite MG, Feuerwerker LCM, Tanaka OY, Duarte L, Weiller JAB, Lara NCC, Botelho LAM, Almeida CAL. O processo de construção da gestão regional da saúde no estado de São Paulo: subsídios para análise. Saude Soc 2015; 24(2):423-436.

36. Casanova AO, Cruz MM, Giovanella L, Alves GR, Cardoso GCP. A implementação de redes de atenção e os desafios da governança regional em saúde na Amazônia Legal. Cien Saude Colet 2017; 22(4):1209-1224.

37. Nobre CLC, Oliveira A, Joia JH, Cruz LS, Franco L, Oliveira KRF, Eleuterio J, Rezende ML, Rocha SR. O trabalho em rede nos casos de alta complexidade e de alta vulnerabilidade: a experiência de uma UBS. Disturb Comun 2017; 29(2):377-384.

38. Machado SS, Moreira LCH, Nascimento MAA, Casotti E. Apoio Institucional na ótica de gestores, apoiadores e trabalhadores: uma aproximação da realidade a partir de diferentes lugares. Interface (Botucatu) 2018; 22(66):813-825.

39. Medeiros RHA. Uma noção de matriciamento que merece ser resgatada para o encontro colaborativo entre equipes de saúde e serviços no SUS. Physis 2015; 25(4):1165-1184.

40. Viana ALD, Bousquat A, Melo GA, Negri Filho A, Medina MG. Regionalização e Redes de Saúde. Cien Saude Colet 2018; 23(6):1791-1798.

41. Velho APM, Vermelho SCSD, Lucena TFR, Bortolozzi F, Bennemann RM. Estratégias de comunicação das Secretarias Municipais de Saúde: desafios para as Redes de Atenção à Saúde. Saude Pesqui 2018; 11(3):505515.

Artigo apresentado em 31/03/2020

Aprovado em 28/08/2020

Versão final apresentada em 30/08/2020

Editores-chefes: Romeu Gomes, Antônio Augusto Moura da Silva 